\title{
An Extension of Newman-Janis algorithm for Rotation Metrics in General Relativity
}

\author{
Yu-Ching Chou M.D.
}

Received: date / Accepted: date

\begin{abstract}
The Newman-Janis algorithm is widely known in the solution of rotating black holes in general relativity. By means of complex transformation, the solution of the rotating black hole can be obtained from the seed metric of a static black hole. This study shows that the extended Newman-Janis algorithm must treat the tensor structure and the seed metric function separately. In the tensor structure, there are two prescriptions, the Newman-Penrose null tetrad and the Giampieri prescription. Both are mathematically the same, while the latter is more concise. In the seed metric function, the extended rules of complex transformation are given in the power of $r$, and the formulaic solution is deduced. Some exact solutions are derived by the extended algorithm, including the Kerr metric, the Kerr-Newman metric, the rotating-de Sitter, the Kerr-de Sitter metric, and the Kerr-Newman-de Sitter metric.
\end{abstract}

Keywords Newman-Janis algorithm $\cdot$ Kerr metric $\cdot$ Kerr de Sitter $\cdot$ Kerr-Newman metric $\cdot$ Kerr-Newman de Sitter metric

PACS 04.20.Jb

\section{Introduction}

General relativity is the most successful gravitational theory in modern physics. The core theory is the Einstein field equation, which is a second order hyperbolic partial differential equation with space-time as its independent variables and metric as its dependent variable. The equation is so highly non-linear that it is difficult to find an exact solution. There are different types of solutions to field equations. A black hole is an important object of study in gravitational theory. In addition to the core of galaxies, the number of large-mass black holes in the universe may be more than imagined. For

Health101 Clinic

1F., No.97, Guling St., Zhongzheng Dist., Taipei City 10078, Taiwan

Tel.: +886-933174117

Fax: +886-2-23648027

E-mail: unclejoe0306@gmail.com 
this reason it plays an important role. With all the possible black hole solutions rotating black holes are crucial because astrophysics believes most astrophysical black holes are rotating. These solutions can also provide external metrics for rotating stars.

To discover new solutions to Einstein's field equations, there are several ways to introduce techniques such as Newman-Penrose formalism and Newman-Janis algorithm (NJA) [1], whose direct result is the Kerr-Newman metric. In recent years, several works have focused on NJA, which is considered as the generation technique of field equations. It is a powerful tool that can generate the axi-symmetric solution of rotating black hole from the corresponding seed metric possessing spherical symmetry. As the previous study proved that the black hole solution that can be generalized by the NJA is Petrov type D classification [2]. However, some literature indicates that NJA can also produce exact solutions of Petrov II fluid [3].

Generally, NJA needs to perform a complex transformation with Newman-Penrose formalism and null tetrad. The Newman-Penrose (NP) spin coefficients, Ricci-NP scalars, and Weyl scalars can be given, which are useful for solving unknown new analytical solutions. The literature points out that NJA can also be performed with Giampieri prescription, which is mathematically equivalent to NP null tetrad [4], and also provides the metric directly without lengthy calculations.

NJA was later developed by Ibohal et al., and used to solve more generalized nonstationary rotational black holes, such as the Vaidya-Kerr-Newman de Sitter metrics [3], and the variable cosmological constant function $\Lambda(u)$ to find a non-stationary de-Sitter cosmological model [5]. In recent years, Erbin further extended NJA to the rotation solution of a NUT charged black hole [6]. However, the generalization rules of NJA is ambiguous on $r^{2}$ or higher order terms in seed metric function.

The aim of this research is to extend the formula of NJA, and to discover the rules of complexifying seed metric. The extension of NJA can help to determine more generalized axi-symmetric solutions in general relativity.

The rest of this study is summarized as follows: Section 2 presents the general procedures of NJA. Section 3 presents the extended formula of NJA on the basis of seed metric function and analyze this technique to various axi-symmetric solutions in general relativity. Section 4 presents the Kerr metric, the Kerr-Newman metric, the Kerr-de Sitter metric, and the Kerr-Newman-de Sitter metric via this extended formula of NJA. Section 5, presents the tensor fields. Section 6 presents the discussions.

\section{Newman-Janis algorithm}

The general procedure for the NJA can be summarized as follows:

1. Determines the "seed metric", and uses Eddington-Finkelstein coordinates $(u, r, \theta, \phi)$ to define the seed metric function $f(r)$ 
2. Performs complex coordinate transformations and the corresponding transformations:

(1) Coordinate tensor structure transformation: Tensor structure: ie, $d x_{\mu}$ (two prescriptions: Newman-Janis [1] and Giampieri [3]);

(2) Complexifies the seed metric function: The function $f(r)$ in the real domain is replaced by the complex function $\widetilde{f}(r, \bar{r})$

3. Changes coordinates according to the study's purpose, such as Boyer-Lindquist coordinates or ellipsoid orthogonal coordinates.

\section{Extension formula of Newman-Janis algorithm}

The static spherical symmetry solution of Einstein's field equations can be expressed as follows

$$
\begin{aligned}
d s^{2} & =f d t^{2}-f^{-1} d r^{2}-r^{2} d \Omega^{2}, \\
d \Omega^{2} & =d \theta^{2}+\sin ^{2} \theta d \phi^{2},
\end{aligned}
$$

where $f$ is the seed metric defined as function $f(r)$.

Step 1: Change the coordinates from $(t, r, \theta, \phi)$ to $(u, r, \theta, \phi)$.

Under the transformation $d t=d u-f^{-1} d r$, Eq.(1) becomes the "outgoing" EddingtonFinkelstein coordinates which is given by

$$
d s^{2}=f d u^{2}+2 d u d r-r^{2} d \Omega^{2}
$$

Step 2: Complex transformation by Newman-Penrose formalism In this step, the tensor structure $d x_{\mu}$ will under complex coordinate transformation either Newman-Janis prescription or Giampieri prescription. Both the final results are the same, however, the latter is more concise.

\section{2-1. Newman-Janis prescription: Newman-Penrose null complex tetrad}

By introducing the formalism of null tetrad, the contravariant metric components are written as

$$
g^{\mu v}=l^{\mu} n^{v}+l^{v} n^{\mu}-m^{\mu} \bar{m}^{v}-m^{v} \bar{m}^{\mu},
$$

where the null tetrad vectors $\left(l^{a}, n^{a}, m^{a}, \bar{m}^{a}\right)$ and the gauge field $A^{a}$ are

$$
\begin{aligned}
l^{a} & =\delta_{r}^{a}, \\
n^{a} & =\delta_{u}^{a}-\frac{f}{2} \delta_{r}^{a}, \\
m^{a} & =\frac{1}{\sqrt{2} r}\left(\delta_{\theta}^{a}+\frac{i}{\sin \theta} \delta_{\phi}^{a}\right), \\
\bar{m}^{a} & =\frac{1}{\sqrt{2} r}\left(\delta_{\theta}^{a}-\frac{i}{\sin \theta} \delta_{\phi}^{a}\right), \\
A^{a} & =-f_{A} l^{a} .
\end{aligned}
$$


The matrix form of the metric tensor $g_{\mu v}$ and its inverse matrix $g^{\mu v}$ are given by

$$
\begin{aligned}
g_{\mu \nu} & =\left(\begin{array}{cccc}
f & 1 & 0 & 0 \\
1 & 0 & 0 & 0 \\
0 & 0 & -r^{2} & 0 \\
0 & 0 & 0 & -r^{2} \sin ^{2} \theta
\end{array}\right), \\
g^{\mu \nu} & =\left(\begin{array}{cccc}
0 & 1 & 0 & 0 \\
1 & -f & 0 & 0 \\
0 & 0 & -r^{-2} & 0 \\
0 & 0 & 0 & -r^{-2} \sin ^{-2} \theta
\end{array}\right) .
\end{aligned}
$$

Performs the following complex transformations, $r, u \in R \rightarrow r^{\prime}, u^{\prime} \in C$

$$
r \rightarrow r^{\prime}-i a \cos \theta^{\prime}, u \rightarrow u^{\prime}+i a \cos \theta^{\prime}, \theta \rightarrow \theta^{\prime}, \phi \rightarrow \phi^{\prime}
$$

The transformed tetrads are listed as follow

$$
\begin{aligned}
l^{\prime a} & =\delta_{r^{\prime}}^{a}, \\
n^{\prime a} & =\delta_{u^{\prime}}^{a}-\frac{\widetilde{f}}{2} \delta_{r^{\prime}}^{a}, \\
m^{\prime a} & =\frac{1}{\sqrt{2}\left(r^{\prime}+i a \cos \theta^{\prime}\right)}\left(\delta_{\theta^{\prime}}^{a}+\frac{i}{\sin \theta^{\prime}} \delta_{\phi^{\prime}}^{a}-\left(\delta_{u^{\prime}}^{a}-\delta_{r^{\prime}}^{a}\right) i a \sin \theta^{\prime}\right), \\
\bar{m}^{\prime} & =\frac{1}{\sqrt{2}\left(r^{\prime}-i a \cos \theta^{\prime}\right)}\left(\delta_{\theta^{\prime}}^{a}-\frac{i}{\sin \theta^{\prime}} \delta_{\phi^{\prime}}^{a}+\left(\delta_{u^{\prime}}^{a}-\delta_{r^{\prime}}^{a}\right) i a \sin \theta^{\prime}\right), \\
A^{\prime a} & =-\widetilde{f}_{A}\left(d u^{\prime}-a \sin ^{2} \theta d \phi\right) .
\end{aligned}
$$

Replacing the seed metric function $f(r) \in R$ in the real domain with the complex function $\widetilde{f}(r, \bar{r})$

$$
r \rightarrow r^{\prime}-i a \cos \theta^{\prime}, \bar{r} \rightarrow r^{\prime}+i a \cos \theta^{\prime},|r|^{2}=r \bar{r}=\Sigma
$$

We discover the complex transformed "seed metric" function: $\widetilde{f}(r, \bar{r})$ can be written as the following rule. Removing the prime notation, we have

$$
\widetilde{f}(r, \bar{r})=1-\frac{2 m(r)}{r}\left(\frac{r^{2}}{\Sigma}\right),
$$

where $m(r)$ denotes the mass function as a function of $r$. The mass function can be a linear superposition of mass $M$, charge $Q$, and cosmological constants $\Lambda$, such as

$$
m(r)=M-\frac{Q^{2}}{2 r}+\frac{\Lambda r^{3}}{6}
$$


After complex transformation $g^{\mu v} \rightarrow \widetilde{g}^{\mu v}$ and its inverse matrix $\widetilde{g}_{\mu v}$ are given by

$$
\begin{aligned}
& \widetilde{g}^{\mu v}=\left(\begin{array}{cccc}
-\frac{a^{2} \sin ^{2} \theta}{\Sigma} & 1+\frac{a^{2} \sin ^{2} \theta}{\Sigma} & 0 & -\frac{a}{\Sigma} \\
1 & -\widetilde{f} & 0 & \frac{a}{\Sigma} \\
1+\frac{a^{2} \sin ^{2} \theta}{\Sigma} & 0 & -\frac{1}{\Sigma} & 0 \\
-\frac{a}{\Sigma} & \frac{a}{\Sigma} & 0 & -\frac{1}{\Sigma \sin ^{2} \theta}
\end{array}\right), \\
& \widetilde{g}_{\mu \nu}=\left(\begin{array}{cccc}
\widetilde{f} & 1 & 0 & a \sin ^{2} \theta(1-\widetilde{f}) \\
1 & 0 & 0 & -a \sin ^{2} \theta \\
0 & 0 & -\Sigma & 0 \\
a \sin ^{2} \theta(1-\widetilde{f}) & -a \sin ^{2} \theta & 0 & -\sin ^{2} \theta\left[\left(r^{\prime 2}+a^{2}\right)+a^{2} \sin ^{2} \theta(1-\widetilde{f})\right]
\end{array}\right) .
\end{aligned}
$$

where the line elements read

$$
\begin{aligned}
d s^{2}= & \widetilde{f} d u^{\prime 2}+2 d u^{\prime} d r^{\prime}+2 a \sin ^{2} \theta(1-\widetilde{f}) d u^{\prime} d \phi^{\prime}-2 a \sin ^{2} \theta d r^{\prime} d \phi^{\prime} \\
& -\Sigma d \theta^{2}-\sin ^{2} \theta\left[\left(r^{\prime 2}+a^{2}\right)+a^{2} \sin ^{2} \theta(1-\widetilde{f})\right] d \phi^{\prime 2}
\end{aligned}
$$

2-2. Giampieri prescripation Now we will introduce another transformation method, and the complex transformed metric can be given directly from the EddingtonFinkelstein coordinate in Eq. (2). The Infinitesimal transformation are

$$
\begin{aligned}
& d r \rightarrow d r^{\prime}+a \sin ^{2} \theta d \phi^{\prime}, \\
& d u \rightarrow d u^{\prime}-a \sin ^{2} \theta d \phi^{\prime} .
\end{aligned}
$$

Compares to the Newman-Penrose prescription:

$$
\begin{aligned}
& d r \rightarrow d r^{\prime}+i a \sin \theta d \theta^{\prime}, \\
& d u \rightarrow d u^{\prime}-i a \sin \theta d \theta^{\prime} .
\end{aligned}
$$

Therefore, $i d \theta=\sin \theta d \phi$ is the crucial rule of this prescription, and it can be proved that the result is exactly the same. After the above complex transformations, the Eq. (2) is transformed as follows

$$
\begin{aligned}
d s^{2}= & \widetilde{f}\left(d u^{\prime}-a \sin ^{2} \theta d \phi^{\prime}\right)^{2}+2\left(d u^{\prime}-a \sin ^{2} \theta d \phi^{\prime}\right)\left(d r^{\prime}+a \sin ^{2} \theta d \phi^{\prime}\right) \\
& -\Sigma\left(d \theta^{\prime 2}+\sin ^{2} \theta d \phi^{\prime 2}\right)
\end{aligned}
$$

By simple algebraic calculation, it can be proven that Eq. (15) and Eq. (12) are identical. The lengthy calculations of null tetrad transformation are not performed in this prescription, therefore this prescription is more concise.

\section{Step 3: Change the coordinates}

Example 1: Boyer-Lindquist coordinates $\left(t_{B L}, r_{B L}, \theta_{B L}, \phi_{B L}\right)$ According to Erbin [6], we have

$$
\begin{gathered}
d u^{\prime}=d t_{B L}+g(r) d r^{\prime} \\
d \phi^{\prime}=d \phi_{B L}+h(r) d r^{\prime}
\end{gathered}
$$


Substituting into Eq. (12), solving $g_{t r}=g_{r \phi}=0$, then we obtain

$$
g(r)=-\frac{r^{2}+a^{2}}{\Delta} h(r)=-\frac{a}{\Delta}
$$

where $\Delta=\widetilde{f} \Sigma+a^{2} \sin ^{2} \theta$, then performs the following coordinate transformations

$$
\begin{aligned}
d u^{\prime} & =d t_{B L}-\frac{r^{2}+a^{2}}{\Delta} d r^{\prime}, \\
d \phi^{\prime} & =d \phi_{B L}-\frac{a}{\Delta} d r^{\prime}, \\
d r^{\prime} & =d r_{B L}, \\
d \theta^{\prime} & =d \theta_{B L} .
\end{aligned}
$$

Eq. (11) is further rewritten as Boyer-Lindquist coordinates, and the associated metric line elements and gauge field are listed as follows

$$
\begin{aligned}
d s^{2} & =\widetilde{f} d t_{B L}^{2}-\frac{\Sigma}{\Delta} d r_{B L}^{2}-X \sin ^{2} \theta, d \phi_{B L}^{2}-2 a(\widetilde{f}-1) \sin ^{2} \theta d t_{B L} d \phi_{B L}, \\
X & =r^{\prime 2}+a^{2}+a g_{t \phi}=r^{2}+a^{2}+(1-\widetilde{f}) a^{2} \sin ^{2} \theta, \\
\frac{\Sigma}{\Delta} & =g(r)-a \sin ^{2} \theta h(r), \\
\Delta & =\widetilde{f} \Sigma+a^{2} \sin ^{2} \theta \\
A & =\widetilde{f}_{A}\left(d t_{B L}-a \sin ^{2} \theta d \phi_{B L}\right) .
\end{aligned}
$$

Example 2: ellipsoid coordinate system $(T, r, \theta, \Phi)$

Our previous research has shown that the Kerr metric and the Kerr-Newman metric can be derived after ellipsoid coordinate transformation as follows [7,8]

$$
\begin{aligned}
d T & =d t-a \sin ^{2} \theta d \phi, \\
d \Phi & =d \phi-\frac{a}{r^{2}+a^{2}} d t .
\end{aligned}
$$

Substituting into Eq.(19), then we have

$$
\begin{aligned}
d s^{2} & =\frac{\Delta}{\Sigma} d T^{2}-\frac{\Sigma}{\Delta} d r^{2}-\Sigma d \theta^{2}-\frac{\left(r^{2}+a^{2}\right)^{2} \sin ^{2} \theta}{\Sigma} d \Phi^{2} \\
\Delta & =\widetilde{f} \Sigma+a^{2} \sin ^{2} \theta \\
A & =\widetilde{f}_{A} d T
\end{aligned}
$$

From the above discussion, we complete the extension of NJA, where metric (12) is in Eddington-Finkelstein coordinates, metric (19) is in Boyer-Lindquist coordinates, and metric (21) is in the ellipsoid orthogonal coordinates. We will introduce the usefulness of these metrics in the next section. 


\section{Newman-Janis algorithm in general relativity: Applications}

\subsection{Flat Space}

Applying NJA to the Minkowski space-time, and let $f=1$. The final result is from the spherical polar coordinate (22) to the ellipsoid coordinates (23)

$$
\begin{gathered}
d s^{2}=d t^{2}-d r^{2}-r^{2} d \theta^{2}-r^{2} \sin ^{2} \theta d \phi^{2} \\
d s^{2}=d t^{2}-\frac{\Sigma}{r^{2}+a^{2}} d r^{2}-\Sigma d \theta^{2}-\left(r^{2}+a^{2}\right) \sin ^{2} \theta d \phi^{2} .
\end{gathered}
$$

This result is an important check of consistency, which will help us to extend the algorithm to more general cases.

\subsection{Kerr Metric}

The Schwarzschild metric is a vacuum static spherical symmetric solution in generalized relativity that can be used as a seed metric function.

$$
f_{S}(r)=1-\frac{2 m(r)}{r}, m(r)=M,
$$

The complex transformation is performed according to the rule of Eq. (9)

$$
\begin{aligned}
\widetilde{f}_{K}(r, \bar{r}) & =1-\frac{2 M}{r}\left(\frac{r^{2}}{\Sigma}\right)=1-\frac{2 M r^{\prime}}{\Sigma}, \\
X & =r^{\prime 2}+a^{2}+\frac{2 M r^{\prime}}{\Sigma} a^{2} \sin ^{2} \theta, \\
\Delta_{K}(r) & =\widetilde{f} \Sigma+a^{2} \sin ^{2} \theta=r^{\prime 2}+a^{2}-2 M r^{\prime} .
\end{aligned}
$$

Substituting all the functions of Eq.(25) into the Boyer-Lindquist coordinates of Eq.(19), the Kerr metric-BL form is obtained as follows

$$
\begin{aligned}
d s^{2}= & \left(1-\frac{2 M r}{\Sigma}\right) d t^{2}+\frac{4 M r a \sin ^{2} \theta}{\Sigma} d t d \phi-\frac{\Sigma}{\Delta} d r^{2} \\
& -\Sigma d \theta^{2}-\left(r^{2}+a^{2}+\frac{2 M r a^{2} \sin ^{2} \theta}{\Sigma}\right) \sin ^{2} \theta d \phi^{2}
\end{aligned}
$$

Then substituting into the ellipsoid orthogonal coordinates of Eq.(21), the Kerr metricellipsoid coordinate form is given by.

$$
d s^{2}=\frac{\Delta_{K}}{\Sigma} d T^{2}-\frac{\Sigma}{\Delta_{K}} d r^{2}-\Sigma d \theta^{2}-\frac{\left(r^{2}+a^{2}\right)^{2} \sin ^{2} \theta}{\Sigma} d \Phi^{2} .
$$

These derivation recoveries the Kerr metric. Obviously, when $a$ vanishes, metrics (26) and (27) will return to the Schwarzchild metric. 


\subsection{Kerr-Newman Metric}

The seed metric function is the Reissner-Nordström metric, and the corresponding gauge field is

$$
f_{R N}(r)=1-\frac{2 m(r)}{r}, m(r)=M-\frac{Q^{2}}{2 r}, f_{A}=\frac{Q}{r} .
$$

Complex transformation is performed according to the rule of Eq. (9), and the transformed complex functions are given by

$$
\begin{aligned}
\widetilde{f}_{K N}(r, \bar{r}) & =1-\frac{2 M r^{\prime}-Q^{2}}{\Sigma}, \\
\widetilde{f}_{A} & =\frac{Q}{r}\left(\frac{r^{2}}{\Sigma}\right)=\frac{Q r^{\prime}}{\Sigma}, \\
A & =\widetilde{f}_{A}\left(d t-a \sin ^{2} \theta d \phi\right), \\
X & =r^{\prime 2}+a^{2}+\frac{2 M r^{\prime}-Q^{2}}{\Sigma} a^{2} \sin ^{2} \theta, \\
\Delta_{K N}(r) & =\widetilde{f} \Sigma+a^{2} \sin ^{2} \theta=r^{\prime 2}+a^{2}-2 M r^{\prime}+Q^{2} .
\end{aligned}
$$

Substituting Eq. (29) into Eq.(19), the ellipsoid form of Kerr-Newman metric is given by

$$
d s^{2}=\frac{\Delta_{K N}}{\Sigma} d T^{2}-\frac{\Sigma}{\Delta_{K N}} d r^{2}-\Sigma d \theta^{2}-\frac{\left(r^{2}+a^{2}\right)^{2} \sin ^{2} \theta}{\Sigma} d \Phi^{2} .
$$

When $Q$ gose to zero, metrics (29) and (30) will return to the Kerr metric.

\subsection{Rotating-de Sitter Metric}

An exact solution of the vacuum Einstein equations with a cosmological constant is the de Sitter metric, and Eq.(31) is the seed metric function.

$$
f_{\Lambda}(r)=1-\frac{2 m(r)}{r}, m(r)=\frac{\Lambda r^{3}}{6},
$$

About $r^{2}$ item, Erbin suggest the transformation rule $r^{2} \rightarrow \Sigma[6]$. However, Canonico et al. shows that if the function $\frac{\Lambda r^{2}}{3}$ is converted to $\frac{\Lambda \Sigma}{3}$, the correct Rotating-de Sitter metric could not be obtained by NJA [9]. To avoid this error, Ibohal's study applied Wang-Wu function [4]. In this study we use the rules in Eq.(9) to perform this complex transformation. The results are listed as follows

$$
\begin{aligned}
\widetilde{f}_{R \Lambda}(r, \bar{r}) & =1-\frac{\Lambda r^{\prime 4}}{3 \Sigma}, \\
X & =r^{\prime 2}+a^{2}+\frac{\Lambda r^{\prime 4}}{3 \Sigma} a^{2} \sin ^{2} \theta, \\
\Delta_{R \Lambda}(r) & =\widetilde{f} \Sigma+a^{2} \sin ^{2} \theta=r^{\prime 2}+a^{2}-\frac{\Lambda r^{\prime 4}}{3} .
\end{aligned}
$$


Substituting all the functions of Eq.(32) into Eq.(19), then the Rotating-de Sitter metric in ellipsoid coordinate is obtained by

$$
d s^{2}=\frac{\Delta_{R \Lambda}}{\Sigma} d T^{2}-\frac{\Sigma}{\Delta_{R \Lambda}} d r^{2}-\Sigma d \theta^{2}-\frac{\left(r^{2}+a^{2}\right)^{2} \sin ^{2} \theta}{\Sigma} d \Phi^{2} .
$$

\subsection{Kerr-de Sitter Metric}

In general relativity, the Schwarzschild-de Sitter metric describes a black hole in a de Sitter space with a cosmologic constant. It can be used as a seed metric equation as

$$
f_{S \Lambda}(r)=1-\frac{2 m(r)}{r}, m(r)=M-\frac{\Lambda r^{3}}{6}
$$

Using the rules of Eq.(9), performing complex transformation, then we have

$$
\begin{aligned}
\widetilde{f}_{K \Lambda}(r, \bar{r}) & =1-\frac{2 M r^{\prime}}{\Sigma}-\frac{\Lambda r^{\prime 4}}{3 \Sigma}, \\
X & =r^{\prime 2}+a^{2}+\frac{2 M r^{\prime}+\frac{\Lambda r^{\prime 4}}{3}}{\Sigma} a^{2} \sin ^{2} \theta, \\
\Delta_{K \Lambda}(r) & =\widetilde{f} \Sigma+a^{2} \sin ^{2} \theta=r^{\prime 2}+a^{2}-2 M r^{\prime}-\frac{\Lambda r^{\prime 4}}{3} .
\end{aligned}
$$

Substituting all the functions of Eq.(36) into Eq.(19), we obtain the Kerr-de Sitter metric in ellipsoid form as

$$
d s^{2}=\frac{\Delta_{K \Lambda}}{\Sigma} d T^{2}-\frac{\Sigma}{\Delta_{K \Lambda}} d r^{2}-\Sigma d \theta^{2}-\frac{\left(r^{2}+a^{2}\right)^{2} \sin ^{2} \theta}{\Sigma} d \Phi^{2}
$$

\subsection{Kerr-Newman-de Sitter Metric}

The Reissner-Nordström-de Sitter metric is an exact solution to the Einstein-Maxwell equations in general relativity under a non-vanished cosmological constant $(\Lambda)$, which describes static mass and charge in a vacuum with spherical symmetry. It can be used as a seed metric function, and given by

$$
f_{R N \Lambda}(r)=1-\frac{2 m(r)}{r}, m(r)=M-\frac{Q^{2}}{2 r}+\frac{\Lambda r^{3}}{6}, f_{A}=\frac{Q}{r}
$$


Performing complex transformations via rules of Eq.(9), then we have

$$
\begin{aligned}
\widetilde{f}_{K N \Lambda}(r, \bar{r}) & =1-\frac{2 M r^{\prime}}{\Sigma}+\frac{Q^{2}}{\Sigma}-\frac{\Lambda r^{\prime 4}}{3 \Sigma} \\
\widetilde{f}_{A} & =\frac{Q}{r}\left(\frac{r^{2}}{\Sigma}\right)=\frac{Q r^{\prime}}{\Sigma} \\
A & =\widetilde{f}_{A}\left(d t-a \sin ^{2} \theta d \phi\right), \\
X & =r^{\prime 2}+a^{2}+\frac{2 M r^{\prime}-Q^{2}+\frac{\Lambda r^{\prime 4}}{2}}{\Sigma} a^{2} \sin ^{2} \theta, \\
\Delta_{K N \Lambda}(r) & =\widetilde{f} \Sigma+a^{2} \sin ^{2} \theta=r^{\prime 2}+a^{2}-2 M r^{\prime}+Q^{2}-\frac{\Lambda r^{\prime 4}}{3} .
\end{aligned}
$$

For the derivation of later sections, we substitute all the functions of Eq.(38) into Eq.(19), then the Kerr-Newman-de Sitter metric tensor in BL coordinates is given by

$$
g_{\mu \nu}=\left(\begin{array}{cccc}
\tilde{f} & 0 & 0 & \frac{a \sin ^{2} \theta\left(r^{2}+a^{2}-\Delta\right)}{\Sigma} \\
0 & -\frac{\Sigma}{\Delta} & 0 & 0 \\
0 & 0 & -\Sigma & 0 \\
\frac{a \sin ^{2} \theta\left(r^{\prime 2}+a^{2}-\Delta\right)}{\Sigma} & 0 & 0 & -\sin ^{2} \theta\left[\left(r^{\prime 2}+a^{2}\right)+a^{2} \sin ^{2} \theta(1-\widetilde{f})\right]
\end{array}\right) .
$$

and the inverse matrix tensor is

$$
g^{\mu \nu}=\left(\begin{array}{cccc}
\frac{\left[\left(r^{2}+a^{2}\right)+a^{2} \sin ^{2} \theta(1-\tilde{f})\right]}{\Delta} & 0 & 0 & \frac{a(1-\tilde{f})}{\Delta} \\
0 & -\frac{\Delta}{\Sigma} & 0 & 0 \\
0 & 0 & -\frac{1}{\Sigma} & 0 \\
\frac{a(1-\tilde{f})}{\Delta} & 0 & 0 & -\frac{\widetilde{f}}{\Delta \sin ^{2} \theta}
\end{array}\right)
$$

All the above discussed metrics and related physical features $(\Lambda, a, M, Q)$ are summarized in Table 1.

\section{Tensor Field}

\subsection{Maxwell tensor}

Keane showed the extended NJA can be performed to obtain the Newman-Penrose scalar fields in rotating metrics [10]. However, we will not further extended this method to de-Sitter spacetime due to inconsistent transformation rules. To obtain the Maxwell tensor, we can straightforward begin with the definition. In this section, we use the BL coordinates $(t, r, \theta, \phi)$, and we have

$$
\begin{aligned}
A_{\mu} & =\frac{Q r}{\Sigma}\left(d t-a \sin ^{2} \theta d \phi\right), \\
F_{\mu v} & =\partial_{\mu} A_{v}-\partial_{v} A_{\mu}, \\
F^{\mu v} & =g^{\mu \rho} g^{v \sigma} F_{\mu v} .
\end{aligned}
$$


Then, the Maxwell fields strength tensor associated with the Kerr-Newman-de Sitter solution can be determined as follows

$$
F_{\mu \nu}=\frac{Q}{\Sigma^{2}}\left(\begin{array}{cccc}
0 & 2 r^{2}-\Sigma & -a^{2} r \sin 2 \theta & 0 \\
-\left(2 r^{2}-\Sigma\right) & 0 & 0 & a \sin ^{2} \theta\left(2 r^{2}-\Sigma\right) \\
a^{2} r \sin 2 \theta & 0 & 0 & -a r \sin 2 \theta\left(r^{2}+a^{2}\right) \\
0 & -a \sin ^{2} \theta\left(2 r^{2}-\Sigma\right) & a r \sin 2 \theta\left(r^{2}+a^{2}\right) & 0
\end{array}\right),
$$

and the inverse matrix

$$
F^{\mu v}=\frac{Q}{\Sigma^{3}}\left(\begin{array}{cccc}
0 & -\left(2 r^{2}-\Sigma\right)\left(r^{2}+a^{2}\right) & a^{2} r \sin 2 \theta & 0 \\
\left(2 r^{2}-\Sigma\right)\left(r^{2}+a^{2}\right) & 0 & 0 & a\left(2 r^{2}-\Sigma\right) \\
-a^{2} r \sin 2 \theta & 0 & 0 & -\frac{a r \sin 2 \theta}{\sin ^{2} \theta} \\
0 & -a\left(2 r^{2}-\Sigma\right) & \frac{a r \sin 2 \theta}{\sin ^{2} \theta} & 0
\end{array}\right)
$$

\subsection{Energy momentum tensor}

Using the above Maxwell tensor and metric tensor, we can further calculate the electromagnetic part of the energy momentum tensor, which is given by

$$
\begin{aligned}
T_{\mu \nu} & =\frac{1}{4 \pi}\left(F_{\mu \rho} F_{v}^{\rho}+\frac{1}{4} g_{\mu \nu} F_{\rho \sigma} F^{\rho \sigma}\right) \\
& =\frac{Q^{2}}{8 \pi \Sigma}\left(\begin{array}{cccc}
\frac{\Delta+a^{2} \sin ^{2} \theta}{\Sigma^{2}} & 0 & 0 & \frac{-a \sin ^{2} \theta}{\Sigma^{2}} A_{t \phi} \\
0 & -\frac{1}{\Delta} & 0 & 0 \\
0 & 0 & 1 & 0 \\
\frac{-a \sin ^{2} \theta}{\Sigma^{2}} A_{t \phi} & 0 & 0 & A_{\phi \phi}
\end{array}\right), \\
A_{t \phi} & =r^{2}+a^{2}+\Delta, \\
A_{\phi \phi} & =\frac{\sin ^{2} \theta}{\Sigma^{2}}\left[\left(r^{2}+a^{2}\right)^{2}+a^{2} \Delta \sin ^{2} \theta\right] \\
\Delta & =r^{2}+a^{2}-2 M r+Q^{2}-\frac{r^{4} \Lambda}{3} .
\end{aligned}
$$

Obviously, when $Q$ vanishes, the $T_{\mu \nu}=0$, and if $\Lambda=0, a=0, M \neq 0, Q \neq 0$, the energy momentum tensor (44) will return to the Reissner-Nordström's form.

This completes the complex transformation of NJA, first given by Newman and Janis, and we extend the seed metric function, which give more general rotation solutions to Einstein filed equations. The extended NJA complex transformation rules in this study are summarized in Table 2.

\section{Discussion}

NJA is a powerful complex transformation method that can generate the axi-symetric solution from "seed metric". In this study, the complex transformation rules and formulaic solutions of $f(r)$ seed metric function are introduced. In addition, the study 
showed that it is more concise to derive rotation metrics by Giampieri prescription than by Newman-Penrose null tetrad, and the result is mathematically equal.

Secondarily, the NJA procedure must treat the tensor structure and the seed metric functions separately. In the tensor structure, the coordinate transformation is equivalent to the transformation of the Minkovski metric in polar coordinates into ellipsoidal coordinates. Therefore, all the rotation metrics discussed here can be written in a consistent ellipsoidal orthogonal coordinate (19). About the $f(r)$ seed metric function, the rule of Eq.(9) is used to perform the complex transformation, thus the conversion rule is regarded as an crucial role in the extension of NJA. Gonzalez [11] proposed $\frac{\operatorname{Re} r^{n+2}}{\Sigma}$, however, when $n=0,1$ this formula is obviously wrong, so in this study we take $\frac{r^{2}}{\Sigma}$ times mass function $m(r)$, which comes from $\frac{r}{2}\left(\frac{1}{r}+\frac{1}{\bar{r}}\right)$ and the results are fruitful.

By choosing the appropriate "seed metric", the Kerr metric (with mass), KerrNewman metric (with mass and charge) can be derived. Furthermore, in rotating universe with cosmological constants, Kerr-de Sitter metric (with mass) and KerrNewman-de Sitter metric (with mass and charge) are derived by the extended NJA. The results of this research are the same reported by Ibohal [5] and Gonzalez [10], and different from the results of Erbin [6], Carter [12], Gibbons and Hawking [13], Mallett [14] and Koberlein [15].

The ellipsoid form of Kerr-Newman-de Sitter metric is given in Eq.(45). Note that all the rotation metrics obtained by NJA in this study have the same ellipsoid coordinates $(T, r, \theta, \Phi)$ with Eq.(19). The only variable function is the delta function.

$$
\begin{aligned}
d s^{2} & =\frac{\Delta_{K N \Lambda}}{\Sigma} d T^{2}-\frac{\Sigma}{\Delta_{K N \Lambda}} d r^{2}-\Sigma d \theta^{2}-\frac{\left(r^{2}+a^{2}\right)^{2} \sin ^{2} \theta}{\Sigma} d \Phi^{2}, \\
\Delta_{K N \Lambda}(r) & =r^{2}+a^{2}-2 M r+Q^{2}-\frac{\Lambda r^{4}}{3} .
\end{aligned}
$$

This result comes from the same tensor structure transformation and may explain why the Carters form of Kerr-Newman-de Sitter metric as shown in Eq.(45) can not be obtained by this extended NJA. It is easy to check if $\Lambda=0$, both metrics return to Kerr-Newman metric, and if $a=0$, both metrics return to Reissner-Nordström-de Sitter metric.

$$
\begin{aligned}
d s^{2} & =\frac{\Delta_{r}}{\Sigma}\left[\frac{d T}{\Xi}\right]^{2}-\frac{\Sigma}{\Delta_{r}} d r^{2}-\frac{\Sigma}{\Delta_{\theta}} d \theta^{2}-\frac{\left(r^{2}+a^{2}\right)^{2} \sin ^{2} \theta}{\Sigma} \Delta_{\theta}\left[\frac{d \Phi}{\Xi}\right]^{2}, \\
\Delta_{r} & =r^{2}+a^{2}-2 M r+Q^{2}-\frac{\Lambda r^{2}}{3}\left(r^{2}+a^{2}\right), \Delta_{\theta}=1+\frac{\Lambda}{3} a^{2} \cos ^{2} \theta, \\
\Xi & =1+\frac{\Lambda a^{2}}{3} .
\end{aligned}
$$


Table 1 metric and related features

\begin{tabular}{llllll}
\hline Seed Metric & Rotation Metric & $\Lambda$ & $a$ & $M$ & $Q$ \\
\hline Schwarzschild & & 0 & 0 & $\neq 0$ & 0 \\
& Kerr & 0 & $\neq 0$ & $\neq 0$ & 0 \\
\hline \multirow{2}{*}{ Reissner-Nordstrom } & & 0 & 0 & $\neq 0$ & $\neq 0$ \\
& Kerr-Newman & 0 & $\neq 0$ & $\neq 0$ & $\neq 0$ \\
\hline \multirow{2}{*}{ Schwarzschild-de Sitter } & & $\neq 0$ & 0 & $\neq 0$ & 0 \\
& Kerr-de Sitter & $\neq 0$ & $\neq 0$ & $\neq 0$ & 0 \\
\hline Reissner-Nordstrom-de Sitter & & $\neq 0$ & 0 & $\neq 0$ & $\neq 0$ \\
& \multirow{2}{*}{ Kerr-Newman-de Sitter } & $\neq 0$ & $\neq 0$ & $\neq 0$ & $\neq 0$ \\
& & & & &
\end{tabular}

Table 2 the extended NJA complex transformation rules

\begin{tabular}{ll}
\hline Tensor structure & $r \rightarrow r^{\prime}-i a \cos \theta^{\prime}$ \\
& $u \rightarrow u^{\prime}+i a \cos \theta^{\prime}$ \\
& $\theta \rightarrow \theta^{\prime}$ \\
& $\phi \rightarrow \phi^{\prime}$ \\
& $\left(l^{\mu}, n^{\mu}, m^{\mu}, \bar{m}^{\mu}\right) \rightarrow\left(l^{\prime \mu}, n^{\prime \mu}, m^{\prime \mu},{\overline{m^{\prime}}}^{\mu}\right)$ \\
\hline Seed metric $f(r)=1-\frac{2 m(r)}{r}\left(\frac{r^{2}}{\Sigma}\right)$ & $m(r)=M-\frac{Q^{2}}{2 r}+\frac{\Lambda r^{3}}{6}$ \\
\hline
\end{tabular}

\section{Conclusion}

The extended NJA of this study can successfully generate family of rotation solutions to Einstein's filed equations in general relativity. However, it remains unclear if this method could be applied to the interior Kerr solution or other high-dimension rotation metrics which is worth of further study.

Acknowledgements I would like to specially thank C.E.O. Ruby Lin of Health 101 Clinic, Dr. Simon Lin of Academia Sinica, I feel also indebted to my sister, Shanrong, for her advice in the refinement in my English writing.

\section{References}

1. Newman,E.T.,Janis,A.I.,:Note on the Kerr Spinning Particle Metric. J. Math. Phys. 6,915 (1965) doi https://doi.org/10.1063/1.1704350

2. S. P. Drake and P. Szekeres, An Explanation of the Newman-Janis Algorithm. arXiv:gr-qc/9807001 (1998)

3. Ibohal,N.,:Rotating metrics admitting non-perfect fluids in General Relativity. arXiv:gr-qc/0403098 (2004)

4. Giampier,G.,:Introducing Angular Momentum into a Black Hole Using Complex Variables. Gravity Research Foundation (1990)

5. Ibohal,N.:Non-stationary de Sitter cosmological models. Int. J. Mod. Phys. D 18,853-863 (2009)

6. Erbin,H.:Janis-Newman algorithm: generating rotating and NUT charged black holes. Universe 3(1),19 (2017)

7. Chou,Y.:A derivation of the Kerr metric by ellipsoid coordinate transformation. Int. J. Phy. Sci. 12(11),130-136 (2017) doi https://doi.org/10.5897/IJPS2017.4605 
8. Chou,Y.:A derivation of the Kerr-Newman metric using ellipsoid coordinate transformation. Journal of Applied Physical Science International 10(3), 144 (2018). http://www . ikprress .org/index.php/ JAPSI/article/view/3189

9. Canonico,R.,Parisi,L.,Vilasi,G.,:Theoretical Models for Astrophysical Objects and the Newman-Janis Algorithm, (Eleventh International Conference on Geometry, Integrablllty and Quantization June 5-10, 2009)

10. Keane,A.J.:An extension of the Newman-Janis algorithm. arXiv:1407.4478v1 [gr-qc] (2014)

11. Gonzalez,E.J.:Extended Newman-Janis algorithm for rotating and Kerr-Newman de Sitter and anti de Sitter metrics. arXiv:1504.01728 [gr-qc](2015)

12. Carter,B. in Les Astres Occlus ed. by B. DeWitt, C. M. DeWitt,Gordon and Breach, New York, 57-124 (1973)

13. Gibbons,G.W.,Hawking,S.W,:Cosmological event horizons, thermodynamics, and particle creation. Phys. Rev. D 15, 2738-2751 (1977)

14. Mallett,R.L.,:Metric of a rotating radiating charged mass in a de Sitter space. Phys. Lett. A 126,226228 (1988)

15. Koberlain,B.D.,:Rotating, radiating black holes, inflation, and cosmic censorship. Phys. Rev. D 51,6783-6787 (1995) 